Nervenarzt 2010 $\cdot 81: 911-912$

DOI 10.1007/s00115-010-2953-4

Online publiziert: 31. Juli 2010

(c) Springer-Verlag 2010

\author{
W. Wick ${ }^{1} \cdot$ M. Weller ${ }^{2}$ \\ ${ }^{1}$ Abteilung Neuroonkologie, Neurologische Klinik und Nationales Tumorzentrum, \\ Universitätsklinikum Heidelberg \\ 2 Klinik für Neurologie, Universitäts-Spital Zürich
}

\title{
Strukturen neuroonkologischer Wissenschaft und Versorgung
}

Nicht zuletzt durch die Arbeit der Neurologen in der Neuroonkologischen Arbeitsgemeinschaft (NOA) der Deutschen Krebsgesellschaft hat sich die Neuroonkologie zu einer eigenständigen Organtumordisziplin entwickelt. Dieser Entwicklung wurde mit der Einrichtung von bisher drei Abteilungen für Neuroonkologie unterschiedlicher struktureller Anbindung an die Partnerdisziplinen der klinischen Neurowissenschaften in Bonn, Frankfurt und Heidelberg Rechnung getragen. Neben dem berufspolitischen Aspekt steht die NOA seither für anerkannte Leitlinienentwicklung sowie strukturierte neuroonkologische Fortbildung und hat durch ihre weltweit beachteten Phase-II- und -III-Studien [1, 2, 4, 8, 9] ein eigenständiges Markenzeichen entwickelt. Auf dieser Basis sind heute in veränderten Strukturen der klinischen Forschung u. a. durch die Novellierung des Arzneimittelgesetzes (AMG) internationale Studien über die European Organization for Research and Treatment of Cancer (EORTC, www.eortc.be) als kooperative Unternehmungen mit der NOA möglich; gleichzeitig werden kritische PhaseIII-Studien zu neuen Medikamenten mit dem Ziel der Erstzulassung in der Onkologie inzwischen auch in der Neuroonkologie und unter deutscher Beteiligung durchgeführt [7].

Ein erster Wunsch an eine gute Fee für die Neuroonkologie sollte die Übersetzung der erfolgreichen wissenschaftsgetriebenen Studien im Zugang zu neuen, frühen Phase-I/II-Studien in Europa und in Deutschland formulieren. Diese Studien müssten keine opportunistische Beschäftigung mit zufällig verfügbaren Subs- tanzen bedeuten, sondern aufgrund der hohen Qualität grundlegender und translationaler Forschung die gezielte Durchführung von „First-in-man“- und „Firstin-oncology“-Studien u. a. in den aktuell begutachteten onkologischen Spitzenzentren oder den Comprehensive Cancer Centers (CCC). Trotz der Schwerpunktsetzung in mehreren Zentren der Neuroonkologie und der Bedeutung von Studien nicht nur für die Wissenschaft, sondern auch für die Versorgung gerade von Patienten mit sehr seltenen und sehr ernsten Erkrankungen, besteht hier weiterer Entwicklungsbedarf.

In einem zweiten Wunsch geht es also unmittelbar um die Finanzierung klinischer Forschung.

\section{- Um das Momentum der genannten NOA-Studien zu nutzen, müssen wir jetzt aktiv werden.}

Im Gegensatz zur klinischen Forschung, die nicht erst seit der 12. Novelle des AMG retrospektiv und monozentrisch oder aber industrieabhängig durchgeführt werden muss, sind die Strukturen und Fördermaßnahmen grundlegender und translationaler neuroonkologischer Wissenschaft gut und nachprüfbar erfolgreich. Als leuchtendes Beispiel dient das von der Deutschen Krebshilfe über viele Jahre geförderte Deutsche Gliomnetzwerk, das neben einem einzigartigen Fundus an strukturierten klinischen und molekularen Daten v. a. einen Wissenschaftsverbund sowie eine geänderte Forschungskultur einer notwendigerweise gewebebasierten translationalen Forschung in der Neuroonkologie etabliert hat. Neben wichtigen, grundlegende Fragen adressierenden Arbeiten [2, 5, 6] bildet das vorhandene Material einen Fundus für die systematische Bearbeitung und Validierung auch zukünftiger in kleineren Serien erhobener Hypothesen.

Das Hirntumornetz im Nationalen Genomforschungsnetzwerk bildet seit vielen Jahren eine sehr erfolgreiche an das Deutsche Krebsforschungszentrum angegliederte grundlagenwissenschaftliche neuroonkologische Plattform. Der letzte Wunsch an eine gute Fee ist nicht spezifisch für die Neuroonkologie: Auch diese spannende Disziplin benötigt wissenschaftlichen Nachwuchs, der hilft, die inzwischen wissenschaftlich und für die Versorgung der Patienten Früchte tragenden Entwicklungen der letzten 15 Jahre fortzuführen und zu verstetigen.

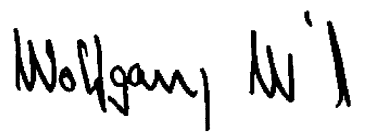

W. Wick

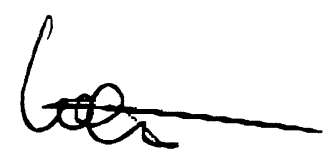

M. Weller

\section{Korrespondenzadresse \\ Prof. Dr. W. Wick}

Abteilung Neuroonkologie, Neurologische

Klinik und Nationales Tumorzentrum,

Universitätsklinikum Heidelberg

Im Neuenheimer Feld 400,

69120 Heidelberg

wolfgang.wick@med.uni-heidelberg.de 


\section{Literatur}

1. Herrlinger U, Schabet M, Brugger W et al (2002) German cancer society neuro-oncology group NOA-03 multicenter trial of single-agent high-dose methotrexate for primary central nervous system lymphoma. Ann Neurol 51:247-252

2. Herrlinger U, Küker W, Uhl M et al (2005) NOA-03 multicenter trial of high-dose methotrexate therapy in primary CNS lymphoma: final report. Ann Neurol 57:843-847

3. Krex D, Klink B, Hartmann C et al (2007) Long-term survival with glioblastoma. Brain 130:2596-2606

4. Neuro-Oncology Working Group (2003) Neuro-Oncology Working Group 01 trial of nimustine plus teniposide versus nimustine plus cytarabine chemotherapy in addition to involved-field radiotherapy in the first-line treatment of malignant glioma. J Clin Oncol 21:3276-3284

5. Weller M, Berger H, Hartmann C et al (2007) Combined $1 p / 19 q$ loss in oligodendroglial tumors: predictive or prognostic biomarker? Clin Cancer Res 13:6933-6937

6. Weller M, Felsberg J, Hartmann C et al (2009) Molecular predictors of progression-free and overall survival in patients with newly diagnosed glioblastoma A prospective translational study of the German Glioma Network. J Clin Oncol 27:5743-5750

7. Wick W, Puduvalli VK, Chamberlain M et al (2010) Enzastaurin (ENZ) versus lomustine (CCNU) in the treatment of recurrent, intracranial glioblastoma: a phase III study. J Clin Oncol 28:1168-1174

8. Wick W, Hartmann C, Engel C et al for the Neurooncology Working Group (NOA) of the German Cancer Society (2009) NOA-04 randomized phase III trial of sequential radiochemotherapy of anaplastic glioma with PCV or temozolomide. J Clin Oncol 27:5874-5880

9. Wick W, Engel C, Combs SE et al for the Neurooncology Working Group (NOA) of the German Cancer Society (2010) NOA-08 randomized phase III trial of one week on/one week off temozolomide versus involved-field radiotherapy in elderly ( $>65$ years) patients with newly diagnosed anaplastic astrocytoma or glioblastoma (Methvsalem). J Clin Oncol (Suppl):7 (Abstr LBA2001)

\section{Studie zur Behandlung des Morbus Menière: Teilnehmer gesucht}

Für eine multizentrische Studie der Universitätsklinik der Ludwig-MaximiliansUniversität München werden Patienten mit Morbus Menière gesucht.

Die Therapie des Morbus Menière stellt noch immer ein großes Problem dar. Zwar existiert eine Fülle von Behandlungsstrategien, jedoch gibt es bisher keine nach „state-of-the-art"-Prinzipien durchgeführten Studien. Frühere Untersuchungen konnten zeigen, dass eine höhere Dosierung von Betahistin therapeutisch wirksamer ist als eine niedrigere.

Ausgehend von diesen Befunden wird nun eine multizentrische, Placebo-kontrollierte, doppelblinde Dosisfindungsstudie durchgeführt. Ziel dieser Studie ist es, den Effekt von unterschiedlich hoch dosiertem Betahistin auf die Anzahl der Schwindelattacken zu prüfen. Untersucht werden soll dabei insbesondere eine höhere Dosierung von Betahistin (3x48 mg pro Tag), die bisher noch nicht zur Behandlung des Morbus Menière zugelassen ist. Die

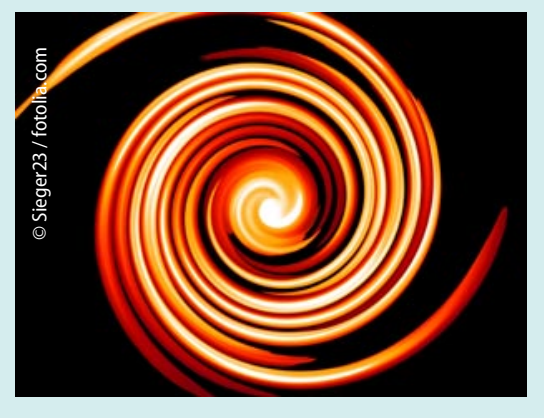

Zehn Zentren in den folgenden Städten beteiligen sich an der Studie: Aachen, Berlin, Erlangen-Nürnberg, Essen, München, Regensburg, Tübingen.
Studie (EudraCT-Nr. 2005-000752-32) wird vom Bundesministerium für Bildung und Forschung finanziert (BMBF177zfyGT).

\section{Rahmendaten der Studie:}

\section{Einschlusskriterien:}

Definitiver M. Menière gemäß den Kriterien der American Academy of Otolaryngology Head and Neck Surgery:

zwei oder mehr Drehschwindelattacken von mindestens 20 Minuten Dauer

— Tinnitus oder Völlegefühl im betroffenen Ohr

- Ausschluss anderer Ursachen

- audiometrisch dokumentierter Hörverlust

- mindestens zwei Menière-Attacken pro Monat für mindestens 3 aufeinander folgende Monate

- Alter: 18-80 Jahre
Für diese klinisch wichtige Behandlungsstudie werden dringend geeignete Patienten/-innen gesucht. Kollegen/-innen, die in Fragekommende Patienten behandeln, können sich direkt bei Herrn Prof. Michael Strupp über die Studie und das für Sie nächstgelegene Studienzentrum informieren. Dies gilt auch für interessierte Patienten.

\section{Ansprechpartner:}

Prof. Dr. Michael Strupp

Klinikum der Universität München, Campus Großhadern

Neurologische Klinik und IFB Schwindel

tel: 089 / 7095 - 6678 oder 6680

fax: 089 / $7095-6673$

E-Mail: Michael.Strupp@med.uni-muenchen.de

Quelle: Ludwig-MaximiliansUniversität München, www.schwindelambulanz-muenchen.de 